\title{
Marine Sediments Harbor Diverse Archaea and Bacteria With Potentials for Anaerobic Hydrocarbon Degradation via Fumarate Addition
}

\section{Chuwen Zhang}

Sun Yat-Sen University

Rainer U. Meckenstock

University of Duisburg-Essen

\section{Shengze Weng}

Sun Yat-Sen University

\section{Guangshan Wei}

Sun Yat-Sen University

\section{Casey R.J. Hubert}

University of Calgary

Jiang-Hai Wang

Sun Yat-Sen University

Xiyang Dong ( $\nabla$ dongxy23@mail.sysu.edu.cn )

Sun Yat-Sen University https://orcid.org/0000-0002-9224-5923

\section{Research}

Keywords: Anaerobic hydrocarbon degradation, Fumarate addition, Metagenome-assembled genomes, Uncultured archaea and bacteria, Marine sediments

Posted Date: September 10th, 2020

DOI: https://doi.org/10.21203/rs.3.rs-71489/v1

License: (c) (1) This work is licensed under a Creative Commons Attribution 4.0 International License. Read Full License 


\section{Abstract}

Background: Marine sediments can contain large amounts of alkanes and methylated aromatic hydrocarbons that are introduced by natural processes or anthropogenic activities. These compounds can be biodegraded by anaerobic microorganisms via enzymatic addition of fumarate. Previous geneand genome-based surveys have detected ubiquitous and novel fumarate-adding enzymes (FAE), but these were neither confirmed as occurring within full degradation pathways nor affiliated with known organisms. The identity and ecological roles of a significant fraction of anaerobic hydrocarbon degraders in marine sediments therefore remains unknown.

Results: By combining phylogenetic reconstructions, protein homolog modelling, and functional profiling of publicly available and newly sequenced metagenomes and genomes, $61 \mathrm{draft}$ bacterial and archaeal genomes encoding anaerobic hydrocarbon degradation via fumarate addition were obtained. Besides Deltaproteobacteria that are well-known to catalyze these reactions, Chloroflexi are dominant FAEencoding bacteria in hydrocarbon-impacted sediments, potentially coupling sulfate reduction or fermentation to anaerobic hydrocarbon degradation. Among Archaea, besides Archaeoglobi previously shown to have this capability, genomes of Heimdallarchaeota, Lokiarchaeota, Thorarchaeota and Thermoplasmata also suggest fermentative hydrocarbon degradation using archaea-type FAE. The biogeography survey reveals these bacterial and archaeal hydrocarbon degraders occur in a wide range of marine sediments, including high abundances of FAE-encoding Asgard archaea associated with natural seeps and subseafloor ecosystems.

Conclusions: Our results expand the knowledge of novel microbial lineages engaged in anaerobic degradation of alkanes and methylated aromatic hydrocarbons, and shed new light on the importance of marine sedimentary archaea in hydrocarbon degradation.

\section{Background}

Marine sediments are the largest reservoir of organic carbon on earth [1], with hydrocarbons constituting a quantitatively important portion of this carbon [2]. Hydrocarbons in marine sediments typically originate from either abiotic thermogenic processes with deep subsurface origins [3] or surface-associated biological processes (e.g., by hydrocarbon-producing alga Botryococcus braunii) [4]. Additionally, anthropogenic activities or accidental oil spills can cause hydrocarbons to settle onto seafloor sediments [5]. Microorganisms inhabiting marine sediments can utilize hydrocarbons as energy and carbon sources in both oxic and anoxic layers of the seabed [6]. In hydrocarbon-impacted sediments, the extra biological oxygen demand usually results in an oxic layer that extends for only the upper few centimeters of the sediment, with anoxic conditions below. Hydrocarbons usually adhere to the sediment matrix and can even block diffusion into the pores at very high hydrocarbon content, which can affect oxygen replenishment [7-10]. Therefore, anaerobic degradation of hydrocarbons is a pivotal process governing the long-term fate of hydrocarbons in marine sediments. 
The strong $\mathrm{C}-\mathrm{H}$ bonds of hydrocarbons pose an energetic and mechanistic challenge for microbial attack [11]. Under oxic conditions, aerobic microorganisms overcome this high energetic barrier using highly reactive oxygen species and oxygenase enzymes [6]. By contrast, anaerobic hydrocarbon degraders employ diverse enzymatic mechanisms to attack these compounds without molecular oxygen as co-substrate. These mechanisms include fumarate addition to alkyl groups, hydroxylation, carboxylation and reverse methanogenesis $[11,12]$. Among these, the most extensively studied and prevalent pathway in most ecosystems is the enzymatic addition of fumarate to hydrocarbon compounds [13]. These enzymes form a sub-branch within the glycyl-radical enzyme superfamily [11]. Hydrocarbon activation mediated by fumarate addition is initiated by generation of a thiyl radical in the active site that abstracts a hydrogen atom from the hydrocarbon [14]. Various clades of fumarate-adding enzymes (FAE) catalyze the activation of different types of hydrocarbon substrates, for example, alkylsuccinate synthases (Ass) for long-chain and short-chain alkanes, benzylsuccinate synthases (Bss) for alkyl-substituted benzenes, and naphthylmethylsuccinate synthases (Nms) for methylnaphthalenes [15-17].

Experimentally validated hydrocarbon-degrading bacteria that use fumarate addition reactions had long been known solely as members of the Proteobacteria and Firmicutes, with notable deltaproteobacterial examples being the sulfate-reducing bacteria Desulfosarcina sp. BuS5, Thauera aromatica and Deltaproteobacterium N47 [17-19]. Metagenomics revealed that Atribacteria from oil reservoirs are also potentially capable of hydrocarbon degradation via fumarate addition [20]. To date, the thermophilic sulfate-reducer Archaeoglobus fulgidus strain VC-16 is the only isolated archaeon known to catalyze anaerobic hydrocarbon biodegradation through addition of fumarate [21]. Metagenomic analysis of a different oil reservoir revealed an Archaeoglobus encoding and expressing the alpha subunit of alkylsuccinate synthase (AssA) [22]. Investigations of marine sediments from cold seeps, hydrothermal fields and deep subsurface layers using genome-resolved metagenomics have revealed that hydrocarbon oxidizers that use fumarate addition might have an even wider phylogenetic distribution [23-26].

Despite these advances in understanding FAE-dependent hydrocarbon degradation, studies so far have either focused only on hydrocarbon-enriched terrestrial environments or on one specific marine ecosystem. As such, the diversity and distribution of FAE-based anaerobic hydrocarbon degraders across sediments of various marine ecosystems remains difficult to ascertain. Ecological studies employing functional gene markers (e.g., assA) demonstrated ubiquitous presence and diversity of anaerobic hydrocarbon degraders in diverse marine environments [27-29]. Nevertheless, further linking functional genes to corresponding microbial taxa and elucidating hydrocarbon degradation pathways is lacking.

Here we aim to (1) identify novel lineages of FAE-harboring hydrocarbon degraders, (2) reconstruct metabolic pathways for hydrocarbon oxidizers identified in this study, and (3) compare distributions of hydrocarbon degraders across different marine ecosystems. To this end, we probed FAE-encoding genomes based on a wide range of metagenomic data derived from sediments of diverse marine ecosystems, including the marine subsurface sediments, natural seeps, hydrothermal fields and oilpolluted sediments. We also examined available archaeal genomes in public databases and linked them 
to marine sediments via genome-wide quantitative read recruitments. Combining these approaches, we report the discovery of novel archaeal and bacterial lineages inhabiting marine sediments capable of anaerobic hydrocarbon degradation via fumarate addition.

\section{Results}

\section{Discovery of novel archaeal and bacterial lineages containing FAE genes}

Ass/Bss/Nms each consists of three subunits and form an $(\alpha \beta \gamma)_{2}$ heterohexamer [21]. Genes encoding the alpha (catalytic) subunits of Ass/Bss/Nms (thereafter referred to as FaeA) are regarded as diagnostic genetic markers for anaerobic hydrocarbon biodegradation [28]. A local database was established $(n=66$; Table S1), that included (1) canonical FaeA protein sequences of Deltaproteobacteria and Firmicutes, and (2) archaea-type AssA protein sequences in Vallitalea guaymasensis strain L81 and A. fulgidus strain VC16. Based on this reference, 65 metagenomic datasets ( 600 gigabase) were probed from a variety of different hydrocarbon-associated marine sediments as well as 3667 archaeal genomes (accessed from NCBI in September 2019) for FaeA protein sequences (Figure S1andTable S2).

Interrogating these genomes for FaeA sequences gave rise to 41 bacterial metagenome-assembled genomes, and 21 archaeal genomes from among the 3667 individual genomes downloaded from NCBI. Of these 62 genomes containing FaeA sequences, 45 had estimated completeness from $70-100 \%$, while the other 17 were $51-70 \%$ complete. Estimated contamination ranged from 0 to $9.95 \%$ (Table S3). As expected, most identified protein sequences affiliated to those from the class Deltaproteobacteria $(n=25$; including orders Desulfobacterales, Syntrophobacterales and Desulfuromonadales) (Figure 1). FaeA sequences were also found in Chloroflexi $(n=15$, class Dehalococcoidia) and the relatively unstudied phylum Candidatus Stahlbacteria $(n=1)$. Within the phylum Euryarchaeota, genes encoding FaeA were not only identified in Archaeoglobi $(\mathrm{n}=8)$ that are known to oxidize alkanes, but also in Thermoplasmata $(n=2)$. Other archaea with these genes belong to the Heimdallarchaeota $(n=2)$, Lokiarchaeota $(n=6)$ and Thorarchaeota $(n=3)$, all within Asgard superphylum (Figure 1). Phylogenetic placements of bacterial and archaeal genomes reported here were also cross-checked using 16S rRNA genes (retrieved in 32 out of 62 genomes), revealing taxonomic profiles consistent with the phylogenomic tree (Data S1).

\section{Classification and verification of identified faeA genes}

Previous studies reported that fae genes can be misannotated as pyruvate formate lyases, and vice versa $[21,30]$. Therefore, manual curation of sequences is necessary for accurate identification of fumarate addition genes. Phylogenetic analysis of protein sequences identified from the 62 genomes along with established reference sequences gave rise to five clusters (Figure 2). Groups I to III contained protein sequences of canonical AssA, BssA and NmsA, respectively, in each case exhibiting high sequence similarity (42-100\%) to experimentally proven hydrocarbon-degrading bacteria [15, 30-32] \{Leuthner, 1998 \#215\} (Figure 2, Table S5). One Desulfuromonadales MAG (GB_003647135) encoded both canonical BssA and NmsA, suggesting its ability to degrade multiple substrates [33]. Group IV sequences clustered 
with neither canonical FaeA nor characterized archaea-type AssA (Figure 2). Phylogenetic separation of these sequences from pyruvate formate lyase protein sequences was evident from them sharing only 20\% amino acid identity, compared to 30-64\% with known FaeA (Figure 2;Table S5). Group IV sequences belonged to genomes assigned to Desulfobacterales, Desulfatiglandales, Syntrophobacterales and Dehalococcoidia. Group V consisted of archaea-type AssA (Figure 2) including AssA protein sequences from anaerobic alkane-degrading $V$. guaymasensis strain L81 and $A$. fulgidus strain VC-16 [21, 34]. In agreement with this, Lokiarchaeota, Heimdallarchaeota, Thermoplasmata AssA protein sequences were closely associated with those in $V$. guaymasensis strain L81, with sequence identities of 32-62\%. Meanwhile, the AssA protein sequences of Archaeoglobi and Thorarchaeota were more similar to that of A. fulgidus strain VC-16 (40-100\% identity) (Table S5). One genome of Candidatus Stahlbacteria phylum also contained a gene encoding for archaea-type AssA (Figure 2).

All recovered protein sequences from Groups I-V were confirmed to be responsible for fumarate addition by analyzing conserved protein motifs. The multiple sequence alignment revealed a conserved glycine residue close to the $\mathrm{C}$-terminus and a conserved cysteine residue in the middle of each aligned protein sequence (Figure S2). This feature has been reported as being specific to FAE, as compared to pyruvate formate lyases that display two adjacent conserved cysteine residues in corresponding regions of protein sequences [30,33]. Apart from the alpha subunit of FAE, genes encoding their accessory beta- and gamma-subunits (FaeB and FaeC) were detected in Deltaproteobacteria and Dehalococcoidia genomes (Figure 3a). Despite the roles of these two small subunits remaining unclear, they are unique to FAE and not found in pyruvate formate lyases [35]. All 21 archaeal genomes analyzed lack genes encoding betaand gamma-subunits, including A. fulgidus VC-16. The same was observed for the short-chain alkane degrader Peptococcaceae strain SCADC [21,31]. It is possible that these shorter sequences were more often missed in lower-coverage genomes or during assembly. Alternatively, given the novelty of Group $\mathrm{V}$ archaea-type Ass, associated small subunits may be more difficult to predict.

Additional structure-based analysis was performed by protein homology modelling. As an example, one Dehalococcoidia MAG ScB_bin257 harbored genes for alpha, beta and gamma subunits of Ass and was screened for modelling. The crystal structure of Bss from Thauera aromatica (PDB ID: 5bwe) agreed with the Ass complex in Dehalococcoidia ScB_bin257. All key residues (i.e. glycine, cysteine, arginine) [36] required for catalytic activities of addition of fumarate to hydrocarbons were observed in the active site of Dehalococcoidia Ass (Figure S3). Other Dehalococcoidia and archaeal genomes were not checked due to absence of beta- and gamma-subunits.

Conversion to the active, radical-containing form of FAE requires an activating enzyme [12,33]. Most of bacterial and archaeal genomes except Heimdallarchaeota contained genes encoding proteins similar to previously reported activating enzymes of FAE with sequence identities of 38-77\% (Figure 3a and Table S6). In addition to the radical SAM cluster binding site in the N-terminus, all activating enzyme sequences described here contained two additional cysteine-rich regions likely involved in iron-sulfur cluster binding (Figure S4). Such a feature is not found in activating enzymes of pyruvate formate lyases $[14,15]$. 


\section{Reconstruction of downstream pathways}

Abilities of bacterial and archaeal lineages to catalyze anaerobic hydrocarbon oxidation was further investigated by assessing the presence of downstream pathways that are required following the addition of fumarate.

Alkylsuccinate metabolism. Following alkane activation, alkylsuccinate is activated to the respective CoA ester followed by carbon-skeleton rearrangement and decarboxylation, yielding branched fatty acids (Figure 4) [37, 38]. These reactions are catalyzed by CoA-ligase (AssK), methylmalonyl-CoA mutase (McmLS) and acetyl-CoA decarboxylase (Acc)/methylmalonyl-CoA decarboxylase (Mcd), respectively. Genes encoding these enzymes were present in most bacterial and archaeal genomes studied here, including Dehalococcoidia $(\mathrm{n}=14)$, Deltaproteobacteria $(\mathrm{n}=18)$, Archaeoglobi $(\mathrm{n}=6)$, Heimdallarchaeota $(n=1)$, Lokiarchaeota $(n=1)$, Thorarchaeota $(n=1)$ and Thermoplasmata $(n=2)$ (Figure $3 \mathbf{b})$. No genes were found to encode enzymes for alkylsuccinate metabolism in the genome of Candidatus Stahlbacteria (Table S7), suggesting its identified Group V FaeA sequence might be contamination arising from misassembly.

Arylalkylsuccinate metabolism. Toluene was considered as a representative alkyl-substituted monocyclic aromatic hydrocarbon. Following initial fumarate addition, the resulting benzylsuccinate is converted to the central intermediate benzoyl-CoA by the multi-subunit enzyme BbsA-H catalyzing several enzymatic steps [33] (Figure 4). Benzoyl-CoA is then dearomatized by BcrA-D/BamB-I and subsequently degraded to CoA-bound fatty acids in modified $\beta$-oxidation reactions by Dch/BamR, Had/BamQ, Oah/BamA [39] (Figure 4). The Bss-containing Desulfuromonadales GB_003647135 had a complete benzoyl-CoA degradation pathway and was regarded as a toluene degrader (Figure 3c). Previous studies suggested that some fumarate addition protein sequences that clustered with NmsA might function in the activation of toluene [40]. Accordingly, NmsA-containing Desulfobacterales (CK_bin19, GB_003646785, SB_bin384) possessed a partial benzylsuccinate degradation pathway. Therefore, these NmsA-containing Desulfobacterales may also be toluene degraders. In addition, Desulfobacterales KS_bin50, which contained Group IV FaeA, also had the potential to oxidize toluene with the support of a near-complete benzylsuccinate degradation pathway (Figure 3c).

For polycyclic aromatic hydrocarbons, 2-methylnaphthalene was used as the representative compound. Following 2-methylnaphthalene activation by fumarate addition, naphthyl-2-methyl-succinate intermediates are converted to 2-naphthoyl-CoA by reactions similar to anaerobic toluene degradation by BnsA-G [41]. However, such genes were not detected in recovered NmsA-containing genomes.

$\beta$-oxidation and fumarate regeneration. After conversion to CoA-bound fatty acids, degradation pathways of aliphatic and aromatic hydrocarbons converge at a conventional $\beta$-oxidation pathway (Figure 4). The resulting fatty acids would be degraded in a series of $\beta$-oxidation reactions (Acd, $C r t$, FadB, and AtoB), ultimately yielding acetyl-CoA [38]. The $\beta$-oxidation pathways were nearly or fully complete in all bacterial and archaeal genomes, with the exception of Candidatus Stahlbacteria (Table S7). In anaerobic alkane degradation, one propionyl-CoA is generated by $\beta$-oxidation (Figure 4) and can be used to recharge the 
fumarate supply. This reaction is catalyzed by a membrane-bound succinate dehydrogenase (Sdh) [38] that was identified in most bacterial and archaeal genomes (Figure 3d).

Mineralization versus fermentation. Hydrocarbons can be either completely mineralized to $\mathrm{CO}_{2}$ or fermented to organic acids and hydrogen as end products of incomplete oxidation. One Dehalococcoidia (EGoM_E44bin31), several Desulfobacterales $(n=8)$ and several Archaeoglobi $(n=5)$ genomes revealed the potential to completely oxidize acetyl-CoA to $\mathrm{CO}_{2}$ via the reverse Wood-Ljungdahl pathway, coupling hydrocarbon oxidation to the sulfate reduction (Figures $3 \mathrm{e}, 4 \mathrm{a}$, and S5-S6). Sulfate-reducing Desulfobacterales also employed a two-step process through acetylphosphate to acetate for energy conservation (Figure 3f) catalyzed by phosphate acetyltransferase (Pta) and acetate kinase (AckA). One Archaeoglobi (Ag-1) carried a gene for respiratory nitrate reductase (NarGH), indicating potential to couple hydrocarbon oxidation with nitrate respiration (Table S4). Additionally, one genome classified as Heimdallarchaeota and eight bacterial genomes contained genes for cytochrome $b d(c y d A B)$, predicted to be involved in protection against $\mathrm{O}_{2}$ to allow strict anaerobes to survive in the presence of nanomolar $\mathrm{O}_{2}$ concentrations [42].

Other Dehalococcoidia $(n=9)$, Syntrophobacterales $(n=3)$, Desulfuromonadales $(n=1)$, Heimdallarchaeota $(n=1)$, Lokiarchaeota $(n=6)$, Thorarchaeota $(n=3)$ and Thermoplasmata $(n=2)$ lacked genes encoding canonical terminal reductases (e.g., ferric iron, sulfate, nitrate). These genomes did contain genes for acetate fermentation catalyzed by ADP-forming acetyl-CoA synthetase (Acs). The fermentation process converted acetyl-CoA to acetate and simultaneously generated ATP via substrate-level phosphorylation (Figures $\mathbf{3 f}$ and $\mathbf{4 b}$ ). Furthermore, genes encoding [FeFe]-hydrogenases and group 4 [NiFe]-hydrogenases for $\mathrm{H}_{2}$ production were also detected in some Dehalococcoidia, Syntrophobacterales,

Desulfuromonadales, Lokiarchaeota, Thermoplasmata and Heimdallarchaeota genomes (Figures $\mathbf{3 f}$ and 4b). These groups might ferment hydrocarbons to reduced products like acetate and hydrogen, similar to scenarios in methanogenic hydrocarbon-degrading enrichment cultures [43].

\section{Environmental distributions of microorganisms containing FAE}

To investigate the biogeography of the newly identified hydrocarbon degraders, their relative abundances were determined in microbial community data derived from 20 geographically different locations (Figure S1 and Table S2). In general, FAE-containing hydrocarbon degraders were broadly distributed in subsurface sediments, natural seeps, hydrothermal fields, and oil-polluted sediments (Figure 5 and Table S8). Hydrocarbon-utilizing taxa were most obviously enriched in natural oil seep sediments, e.g., in the Eastern Gulf of Mexico, Campeche Knolls and Scotain Basin, comprising 10\% of the communities in these environments. Anaerobic hydrocarbon degraders were also present at high abundance in the subsurface sediments not associated with seepage, e.g., accounting for 11 and $16 \%$ of the communities in Costa Rica and Kattegat Sea sediments, respectively. Dehalococcoidia and Deltaproteobacteria were predominant bacterial lineages responsible for anaerobic hydrocarbon degradation in most habitats. Lokiarchaeota comprised relatively large fractions of the communities in both natural oil seeps (up to $6.5 \%$ of the community in Eastern Gulf of Mexico sediments) and the subseafloor (up to $3.8 \%$ of the 
community in Costa Rica). Lokiarchaeota were also found in the sediments from coastal ecosystems, but at low abundances ( $<1 \%$ of community). Hyperthermophilic Archaeoglobi were hardly detected in these marine sediments, even in hydrothermal vent sediments like Guaymas Basin. Recovered 16S rRNA gene sequences from lineages of those anaerobic hydrocarbon-degrading archaea and bacteria were used to search NCBI databases. The result suggested that they might be more prevalent in other environments such as oilfields (Table S9).

\section{Discussion}

Enzymes that catalyze anaerobic hydrocarbon degradation via fumarate addition have been considered to be phylogenetically restricted to a small number of bacterial and archaeal lineages (Deltaproteobacteria, Firmicutes, and Archaeoglobi) [6, 21, 31]. By probing metagenomic data derived from various marine sediment environments and other archaeal genomes, here the diversity of FAE-based hydrocarbon oxidizing microorganisms in domains of both Bacteria and Archaea is extended. Apart from well-documented lineages of Deltaproteobacteria and Archaeoglobi [6, 21], faeA genes are reported here as also occurring in the class Dehalococcoidia within the phylum Chloroflexi, the class Thermoplasmata within the phylum Euryarchaeota, and within the Asgard superphylum (Lokiarchaeota, Thorarchaeota, and Heimdallarchaeota). Current knowledge about archaea involved in anaerobic hydrocarbon degradation focuses mainly on oxidation of methane and other short alkane gases using methyl-CoM reductase or methyl-CoM reductase-like enzymes [25,44,45]. New archaeal diversity presented here for the degradation of longer alkanes opens up a wider perspective on the evolution and expansion of hydrocarbon-oxidizing pathways throughout the archaeal domain. Lab cultivation of members of the bacterial and archaeal groups described here, and enzymatic characterization of the novel fumarateadding enzymes they encode, will enable validation of these metabolic predictions.

Reconstruction of downstream metabolic pathways indicates that different microorganisms have different hydrocarbon substrate preferences. Members of the Chloroflexi, Thermoplasmata and Asgard archaea are genetically capable of oxidizing metabolites produced following initial activation of alkanes, whereas only Desulfobacterales and Desulfuromonadales contained genes involved in arylalkylsuccinate metabolism following activation of alkyl-substituted monocyclic aromatics such as toluene (Fig. 4). This suggests that within microbiomes of different marine sediment environments, a greater diversity of microorganisms are capable of alkane degradation. Aromatic hydrocarbons have to undergo a series of energy-consuming de-aromatization reactions before microbial utilization [11]. The difficulty in breaking down the aromatic ring might explain the discovery of fewer microorganisms capable of aromatic hydrocarbon degradation. Overall, bacteria uncovered here can metabolize a broader range of hydrocarbon substrates than archaea, with the latter only having genes for alkane activation. Deeper investigations into the evolutionary history of bacterial and archaeal FAE should uncover mechanisms that have selected for different hydrocarbon substrates in these microbial groups.

Genomic evidence suggests that fermentative activation of hydrocarbons as part of syntrophic associations, e.g., with sulfate-reducers or methanogens, is an important hydrocarbon degradation 
mechanism in marine sediments. In addition to members of Syntrophobacterales that have been shown to initiate methanogenic hydrocarbon biodegradation [30,46], evidence from the genomes analyzed here reveals that members of Dehalococoidia, Desulfuromonadales, Thermoplasmata and three Asgard members (Lokiarchaeota, Thorarchaeota and Heimdallarchaeota) lack genes for reduction of inorganic electron acceptors. Fermentation is therefore necessary for these organisms to be capable of growth on hydrocarbons. Electrons generated during hydrocarbon oxidation would need to be transferred to syntrophic partners, e.g., as hydrogen or acetate [47]. Such partnerships can maintain energetically favorable concentrations of hydrogen or acetate necessary for syntrophic oxidation of the hydrocarbons.

Bacteria and archaea capable of anaerobic hydrocarbon degradation are widely distributed and are not limited to hydrocarbon-rich environments. It is not surprising that hydrocarbon-degrading microorganisms are most prevalent in sediments featuring natural seepage of oil and gas, due to high concentrations of hydrocarbons at these sites $[23,48,49]$. However, in subsurface sediments that do not feature seepage, relatively high abundances of hydrocarbon degraders are also observed (Fig. 5), although the ecological significance of this capability, and whether these genes are expressed in these settings, requires further investigation. Previous studies often emphasize the roles of bacteria and overlook the role played by archaea during hydrocarbon degradation. This work also shows that in marine sediments Lokiarchaeota are among the most dominant hydrocarbon degraders, for example, comprising up to $6.5 \%$ of the community in seep sediments from Eastern Gulf of Mexico. This points to archaea also playing an essential role in determining the fate of hydrocarbon compounds.

\section{Conclusions}

The phylogenetic range for anaerobic hydrocarbon metabolism mediated by fumarate addition mechanism is much greater than previously reported, with several archaea and bacteria in marine sediments equipped with genetic repertoires for different types of FAE. Most newly identified anaerobic hydrocarbon degraders appear to rely on a fermentative lifestyle. Archaeal members like Lokiarchaeota may be as important as bacteria in anaerobic degradation of longer chain alkanes, especially in areas of natural seeps and subsurface sediments.

\section{Methods}

\section{Establishment of a reference protein database for fumarate-adding enzymes}

FaeA reference protein sequences were collected from Genbank and publicly available microbial isolate genomes and enrichment cultures. Sequences $\leq 700$ amino acids in length were excluded. A phylogenetic tree of retrieved reference protein sequences was built to confirm sequences corresponded to the alpha subunit of fumarate-adding enzymes. This tree was established on IQTree web Server under the TEST option for best model selection with 1000 ultrafast bootstrap replicates [50]. The final reference database comprises 66 full-length FaeA protein sequences (Table S1). 


\section{Data acquisition and initial screen of (meta)genome datasets}

To identify novel lineages of anaerobic hydrocarbon degraders, 65 metagenome datasets derived from various marine sediments were downloaded from NCBI's Sequence Read Archive (https://www.ncbi.nlm.nih.gov/sra/). Metagenomes were categorized based on four representative marine ecosystems: natural seeps, subsurface sediments, hydrothermal fields, and oil-polluted sediments. For each ecosystem, at least five sites were selected (Figure S1 and Table S2). In addition, genome sequences from 3667 Archaea, representing all archaeal genomes present at NCBI as of September 2019, were downloaded and de-replicated using dRep v2.3.2 [51] at 99\% average nucleotide identity.

To screen these metagenome datasets for the presence of genes encoding FaeA, a gene-centric analysis was conducted using GraftM v0.12.2 [52]. First, 'graftM create' was used to generate a GraftM package with FaeA protein sequences and their associated taxonomy (Table S1) as input; then, 'graftM graft' was employed to measure short-read counts of genes encoding FaeA in quality-filtered metagenome reads. Based on gene abundances in each metagenome, 48 datasets with CPM (counts per million reads) of FaeA $>20$ were kept for further analyses (Table S10).

\section{Metagenome assembly and binning}

Metagenomic raw reads were subject to quality control, assembly and binning with the metaWRAP v1.2 pipeline [53]. Briefly, raw reads were trimmed by the metaWRAP Read_qc module with default parameters. Each dataset was assembled using the metaWRAP Assembly module (-megahit). For each assembly, contigs were binned into MAGs with the metaWRAP Binning module (--maxbin2 -metabat1 -metabat2); the three resulting bin sets were then consolidated into a final bin set using the metaWRAP Bin_refinement module (-c $50-x$ 10). All produced MAGs were aggregated and dereplicated with dRep v2.3.2 (-comp 50 -con 10) [51]. Completeness and contamination of MAGs were estimated based on lineage-specific marker genes using CheckM v1.0.13 [54].

\section{Functional annotations}

Gene predictions were undertaken with Prokka v1.14.0 [55]. Functional annotations were performed using GhostKOALA (genus_prokaryotes + family_eukaryotes) [56]. Annotations were also curated by searching against Pfam, TIGRfam and custom HMM databases using MetaErg [57] and METABOLIC [58]. Genes involved in anaerobic hydrocarbon degradation were screened using BLASTp (cutoffs: identity $>30 \%$, coverage $>90 \%, e<1 \times 10^{-20}$ ) against the FaeA reference protein database (Table S1). Hydrogenases were retrieved from genomes through DIAMOND BLASTp v0.9.21.122 [59] queries against comprehensive custom databases [60] (cutoffs: identity $>50 \%, e<1 \times 10^{-20}$ ) and refined using the hydrogenase classifier HydDB [61].

\section{Protein structure modeling}


Tertiary structures of Ass were predicted using SWISS-MODEL [62] with the default parameters. Benzylsuccinate synthase (Bss) from Thauera aromatica [35] was selected as the structural template. PyMOL v1.7.4 (https://pymol.org/2/) was then used to superimpose the modelled structures on the template Bss structure (PDB ID:5BWE) and visualize the active sites of putative Ass.

\section{Conserved residues and motifs}

Conserved residues in recovered FaeA proteins were identified by aligning with reference proteins. MAFFT [63] was used to perform the sequence alignments. Jalview 2.11 [64] was used to view and edit the alignments.

\section{Phylogenetic trees of functional genes}

For FaeA, amino acid sequences were aligned with MUSCLE v3.8.1551 with default settings [65], followed by removal of ambiguous sites using trimAl v1.4.rev15 (-automated1) [66]. Maximum likelihood phylogenies were calculated in IQTree web Server using the best-fit model and 1000 ultrafast bootstrap replicates [50]. For amino acid sequences of DsrA, a maximum-likelihood tree was constructed in MEGA X [67], using the JTT matrix-based model with all sites, bootstrapped with 50 replicates. The resulting tree was visualized using the Interactive Tree Of Life (iTOL) webtool [68].

\section{Taxonomic assignments of MAGs}

Taxonomic classification of each genome was initially conducted using GTDB-Tk against the database release 89 [69]. A phylogenomic tree based on a concatenated alignment of a set of 43 specific singlecopy marker genes was generated using RAxML v8.2.12 (called as: raxmIHPC-HYBRID -f a -n result -s input -c 25 -N 100 -p 12345 -m PROTCATLG -x 12345) [70] and visualized using iTOL [68]. All genomes were placed to appropriate phylogenetic positions according to the NCBI Taxonomy database, taking into account results of both approaches mentioned above.

\section{Calculation of relative abundances}

To determine the relative abundance of each organism in different environmental samples, qualitycontrolled reads for individual metagenomes were first mapped to the set of dereplicated genomes using BamM 'make' (http://ecogenomics.github.io/BamM). Resulting BAM files were used to calculate relative abundances of each genome using CoverM v0.3.1 (https://github.com/wwood/CoverM) with genome mode (-trim-min 0.10 -trim-max 0.90 -min-read-percent-identity 0.95 -min-read-aligned-percent 0.75 ).

\section{Recovery of 16S rRNA gene sequences}

16S rRNA gene sequences were recovered from genomes of newly identified anaerobic hydrocarbon degraders using MetaErg [57]. Sequences longer than $800 \mathrm{bp}$ were then used as inputs to search against $\mathrm{NCBI} \mathrm{nt} / \mathrm{nr}$ database via BLASTn. Hits from each individual BLAST search with $>95 \%$ coverage and $>97 \%$ identity were retained for further analysis of global distributions in the environmental samples. 


\section{Abbreviations}

FAE: fumarate-adding enzyme; Ass: alkylsuccinate synthase; Bss: benzylsuccinate synthase; Nms: naphthylmethylsuccinate synthase; iTOL: Interactive Tree Of Life; CPM: counts per million reads

\section{Declarations}

\section{Acknowledgements}

Not applicable.

\section{Author contributions}

X.D. designed this study. X.D. and C.Z. analyzed data. X.D., C.Z., R.U.M. and G.W. interpreted the data. C.Z. and S.W. prepared the figures. C.R.J.H contributed part of the data. J.H.W. is the supervisor of C.Z.. The manuscript was written by X.D., C.Z. R.U.M., and C.R.J.H, with input from other authors.

\section{Funding}

The work was supported by the National Natural Science Foundation of China (Grant No. 41906076), the Fundamental Research Funds for the Central Universities (Grant No. 19lgpy90), and the Fund of Guangdong Research and Construction of Public Service Abilities (No. 2017B020218004).

\section{Availability of data and material}

All bacterial and archaeal genomes described in this study can be found at figshare

(https://doi.org/10.6084/m9.figshare.12780806). All other data supporting the findings of this study are available within the article and its supplementary information files, or from the corresponding authors upon request.

\section{Ethics approval and consent to participate}

Not applicable.

\section{Consent for publication}

Not applicable.

\section{Competing interest}

The authors declare no conflict of interest.

\section{References}


1. D'Hondt S, Pockalny R, Fulfer VM, Spivack AJ. Subseafloor life and its biogeochemical impacts. Nat Commun 2019;10:3519.

2. Jørgensen BB, Boetius A. Feast and famine - microbial life in the deep-sea bed. Nat Rev Microbiol 2007;5:770-781.

3. Etiope G, Ciccioli P. Earth's degassing: a missing ethane and propane source. Science 2009;323:478.

4. Banerjee A, Sharma R, Chisti Y, Banerjee UC. Botryococcus braunii: a renewable source of hydrocarbons and other chemicals. Crit Rev Biotechnol 2002;22:245-279.

5. Kimes NE, Callaghan AV, Suflita JM, Morris PJ. Microbial transformation of the Deepwater Horizon oil spill-past, present, and future perspectives. Front Microbio/ 2014;5:603.

6. Widdel F, Knittel K, Galushko A. Anaerobic hydrocarbon-degrading microorganisms: an overview. In: Handbook of Hydrocarbon and Lipid Microbiology. Edited by Timmis KN: Springer, Berlin; 2010;19972021.

7. Orsi WD. Ecology and evolution of seafloor and subseafloor microbial communities. Nat Rev Microbiol 2018;16:671-683.

8. Bowles MW, Mogollón JM, Kasten S, Zabel M, Hinrichs K-U. Global rates of marine sulfate reduction and implications for sub-sea-floor metabolic activities. Science 2014;344:889-891.

9. Klamerus-Iwan A, Błońska E, Lasota J, Kalandyk A, Waligórski P. Influence of oil contamination on physical and biological properties of forest soil after chainsaw use. Water, Air, Soil Pollut 2015;226:389-389.

10. Lueders T. The ecology of anaerobic degraders of BTEX hydrocarbons in aquifers. FEMS Microbiol Ecol 2017;93.

11. Boll M, Estelmann S, Heider J. Anaerobic degradation of hydrocarbons: mechanisms of hydrocarbon activation in the absence of oxygen. In: Anaerobic Utilization of Hydrocarbons, Oils, and Lipids, Handbook of Hydrocarbon and Lipid Microbiology. Edited by Boll M: Springer, Cham; 2018;1-27.

12. Callaghan A. Enzymes involved in the anaerobic oxidation of n-alkanes: from methane to long-chain paraffins. Front Microbio/ 2013;4.

13. Aitken CM, Jones DM, Maguire MJ, Gray ND, Sherry A, Bowler BFJ et al. Evidence that crude oil alkane activation proceeds by different mechanisms under sulfate-reducing and methanogenic conditions. Geochim Cosmochim Acta 2013;109:162-174.

14. Shisler KA, Broderick JB. Glycyl radical activating enzymes: structure, mechanism, and substrate interactions. Arch Biochem Biophys 2014;546:64-71.

15. Callaghan AV, Wawrik B, Ní Chadhain SM, Young LY, Zylstra GJ. Anaerobic alkane-degrading strain AK-01 contains two alkylsuccinate synthase genes. Biochem Biophys Res Commun 2008;366:142148.

16. Annweiler E, Materna A, Safinowski M, Kappler A, Richnow HH, Michaelis W et al. Anaerobic degradation of 2-methylnaphthalene by a sulfate-reducing enrichment culture. Appl Environ Microbiol 2000;66:5329-5333. 
17. Leuthner B, Leutwein C, Schulz H, Hörth P, Haehnel W, Schiltz E et al. Biochemical and genetic characterization of benzylsuccinate synthase from Thauera aromatica: a new glycyl radical enzyme catalysing the first step in anaerobic toluene metabolism. Mol Microbiol 1998;28:615-628.

18. Meckenstock RU, Annweiler E, Michaelis W, Richnow HH, Schink B. Anaerobic naphthalene degradation by a sulfate-reducing enrichment culture. Appl Environ Microbio/ 2000;66:2743-2747.

19. Kniemeyer O, Musat F, Sievert SM, Knittel K, Wilkes H, Blumenberg M et al. Anaerobic oxidation of short-chain hydrocarbons by marine sulphate-reducing bacteria. Nature 2007;449:898-901.

20. Liu Y, Qi Z, Shou L, Liu J, Yang S, Gu J et al. Anaerobic hydrocarbon degradation in candidate phylum 'Atribacteria' (JS1) inferred from genomics. ISME J 2019;13:2377-2390.

21. Khelifi N, Amin Ali O, Roche P, Grossi V, Brochier-Armanet C, Valette $O$ et al. Anaerobic oxidation of long-chain $n$-alkanes by the hyperthermophilic sulfate-reducing archaeon, Archaeoglobus fulgidus. ISME J 2014;8:2153.

22. Liu Y, Galzerani DD, Mbadinga SM, Zaramela LS, Gu J, Mu B et al. Metabolic capability and in situ activity of microorganisms in an oil reservoir. Microbiome 2018;6:5.

23. Dong X, Greening C, Rattray JE, Chakraborty A, Chuvochina M, Mayumi D et al. Metabolic potential of uncultured bacteria and archaea associated with petroleum seepage in deep-sea sediments. Nat Commun 2019;10:1816.

24. Farag IF, Biddle JF, Zhao R, Martino AJ, House CH, León-Zayas RI. Metabolic potentials of archaeal lineages resolved from metagenomes of deep Costa Rica sediments. ISME J 2020;14:1345-1358.

25. Dong X, Rattray JE, Campbell DC, Webb J, Chakraborty A, Adebayo $O$ et al. Thermogenic hydrocarbons sustain diverse subseafloor microbial communities in deep sea cold seep sediments. bioRxiv 2020; doi:https://doi.org/10.1101/2020.02.02.928283.

26. Dombrowski N, Seitz KW, Teske AP, Baker BJ. Genomic insights into potential interdependencies in microbial hydrocarbon and nutrient cycling in hydrothermal sediments. Microbiome 2017;5:106.

27. Gittel A, Donhauser J, Røy H, Girguis PR, Jørgensen BB, Kjeldsen KU. Ubiquitous presence and novel diversity of anaerobic alkane degraders in cold marine sediments. Front Microbio/ 2015;6:1414.

28. Stagars MH, Ruff SE, Amann R, Knittel K. High diversity of anaerobic akane-degrading microbial communities in marine seep sediments based on (1-methylalkyl)succinate synthase genes. Front Microbiol 2016;6:1511.

29. Stagars MH, Mishra S, Treude T, Amann R, Knittel K. Microbial community response to simulated petroleum seepage in Caspian Sea sediments. Front Microbiol 2017;8:764.

30. Tan B, Nesbø C, Foght J. Re-analysis of omics data indicates Smithella may degrade alkanes by addition to fumarate under methanogenic conditions. ISME J 2014;8:2353-2356.

31. Tan B, Charchuk R, Li C, Nesbø C, Abu Laban N, Foght J. Draft genome sequence of uncultivated Firmicutes (Peptococcaceae SCADC) single cells sorted from methanogenic alkane-degrading cultures. Genome Announc 2014;2:e00909-00914. 
32. Bergmann F, Selesi D, Weinmaier T, Tischler P, Rattei T, Meckenstock RU. Genomic insights into the metabolic potential of the polycyclic aromatic hydrocarbon degrading sulfate-reducing Deltaproteobacterium N47. Environ Microbiol 2011;13:1125-1137.

33. Heider J, Szaleniec M, Martins BM, Seyhan D, Buckel W, Golding BT. Structure and function of benzylsuccinate synthase and related fumarate-adding glycyl radical enzymes. J Mol Microbiol Biotechnol 2016;26:29-44.

34. Schouw A, Leiknes Eide T, Stokke R, Pedersen RB, Steen IH, Bødtker G. Abyssivirga alkaniphila gen. nov., sp. nov., an alkane-degrading, anaerobic bacterium from a deep-sea hydrothermal vent system, and emended descriptions of Natranaerovirga pectinivora and Natranaerovirga hydrolytica. Int J Syst Evol Microbiol 2016;66:1724-1734.

35. Funk MA, Marsh ENG, Drennan CL. Substrate-bound structures of benzylsuccinate synthase reveal how toluene is activated in anaerobic hydrocarbon degradation. J Biol Chem 2015;290:22398-22408.

36. Funk MA, Judd ET, Marsh ENG, Elliott SJ, Drennan CL. Structures of benzylsuccinate synthase elucidate roles of accessory subunits in glycyl radical enzyme activation and activity. Proc Natl Acad Sci USA 2014;111:10161.

37. Singh R, Guzman MS, Bose A. Anaerobic oxidation of ethane, propane, and butane by marine microbes: a mini review. Front Microbio/ 2017;8:2056.

38. Callaghan AV, Morris BEL, Pereira IAC, Mclnerney MJ, Austin RN, Groves JT et al. The genome sequence of Desulfatibacillum alkenivorans AK-01: a blueprint for anaerobic alkane oxidation. Environ Microbiol 2012;14:101-113.

39. Fuchs G, Boll M, Heider J. Microbial degradation of aromatic compounds - from one strategy to four. Nat Rev Microbiol 2011;9:803-816.

40. Tan B, Jane Fowler S, Laban NA, Dong X, Sensen CW, Foght J et al. Comparative analysis of metagenomes from three methanogenic hydrocarbon-degrading enrichment cultures with 41 environmental samples. ISME J 2015;9:2028-2045.

41. Wilkes $\mathrm{H}$, Rabus R. Catabolic pathways involved in the anaerobic degradation of saturated hydrocarbons. In: Anaerobic Utilization of Hydrocarbons, Oils, and Lipids, Handbook of Hydrocarbon and Lipid Microbiology. Edited by Boll M: Springer, Cham; 2019;1-24.

42. Safarian S, Rajendran C, Müller H, Preu J, Langer JD, Ovchinnikov S et al. Structure of a bd oxidase indicates similar mechanisms for membrane-integrated oxygen reductases. Science 2016;352:583586.

43. Mbadinga SM, Li K, Zhou L, Wang L, Yang S, Liu J et al. Analysis of alkane-dependent methanogenic community derived from production water of a high-temperature petroleum reservoir. App/ Microbiol Biotechnol 2012;96:531-542.

44. Wang Y, Wegener G, Hou J, Wang F, Xiao X. Expanding anaerobic alkane metabolism in the domain of Archaea. Nat Microbiol 2019;4:595-602.

45. Baker BJ, De Anda V, Seitz KW, Dombrowski N, Santoro AE, Lloyd KG. Diversity, ecology and evolution of Archaea. Nat Microbio/ 2020;5:887-900. 
46. Wawrik B, Marks CR, Davidova IA, Mclnerney MJ, Pruitt S, Duncan KE et al. Methanogenic paraffin degradation proceeds via alkane addition to fumarate by 'Smithella' spp. mediated by a syntrophic coupling with hydrogenotrophic methanogens. Environ Microbio/ 2016;18:2604-2619.

47. Gieg LM, Fowler SJ, Berdugo-Clavijo C. Syntrophic biodegradation of hydrocarbon contaminants. Curr Opin Biotechnol 2014;27:21-29.

48. Hawley ER, Malfatti SA, Pagani I, Huntemann M, Chen A, Foster B et al. Metagenomes from two microbial consortia associated with Santa Barbara seep oil. Mar Genomics 2014;18:97-99.

49. Laso-Pérez R, Hahn C, van Vliet DM, Tegetmeyer HE, Schubotz F, Smit NT et al. Anaerobic degradation of non-methane alkanes by "Candidatus Methanoliparia" in hydrocarbon seeps of the Gulf of Mexico. mBio 2019;10:e01814-01819.

50. Trifinopoulos J, Nguyen L-T, von Haeseler A, Minh BQ. W-IQ-TREE: a fast online phylogenetic tool for maximum likelihood analysis. Nucleic Acids Res 2016;44:W232-W235.

51. Olm MR, Brown CT, Brooks B, Banfield JF. dRep: a tool for fast and accurate genomic comparisons that enables improved genome recovery from metagenomes through de-replication. ISME J 2017;11:2864.

52. Boyd JA, Woodcroft BJ, Tyson GW. GraftM: a tool for scalable, phylogenetically informed classification of genes within metagenomes. Nucleic Acids Res 2018;46:e59.

53. Uritskiy GV, DiRuggiero J, Taylor J. MetaWRAP-a flexible pipeline for genome-resolved metagenomic data analysis. Microbiome 2018;6:158.

54. Parks DH, Imelfort M, Skennerton CT, Hugenholtz P, Tyson GW. CheckM: assessing the quality of microbial genomes recovered from isolates, single cells, and metagenomes. Genome Res 2015;25:1043-1055.

55. Seemann T. Prokka: rapid prokaryotic genome annotation. Bioinformatics 2014;30:2068-2069.

56. Kanehisa M, Sato Y, Morishima K. BlastKOALA and GhostKOALA: KEGG tools for functional characterization of genome and metagenome Sequences. J Mol Biol 2016;428:726-731.

57. Dong $X$, Strous M. An integrated pipeline for annotation and visualization of metagenomic contigs. Front Genet 2019;10.

58. Zhou Z, Tran P, Liu Y, Kieft K, Anantharaman K. METABOLIC: a scalable high-throughput metabolic and biogeochemical functional trait profiler based on microbial genomes. bioRxiv 2019; doi:https://doi.org/10.1101/761643.

59. Buchfink B, Xie C, Huson DH. Fast and sensitive protein alignment using DIAMOND. Nat Methods 2015;12:59-60.

60. Greening C, Geier R, Wang C, Woods LC, Morales SE, McDonald MJ et al. Diverse hydrogen production and consumption pathways influence methane production in ruminants. ISME J 2019.

61. Søndergaard D, Pedersen CNS, Greening C. HydDB: a web tool for hydrogenase classification and analysis. Sci Rep 2016;6:34212. 
62. Waterhouse A, Bertoni M, Bienert S, Studer G, Tauriello G, Gumienny R et al. SWISS-MODEL: homology modelling of protein structures and complexes. Nucleic Acids Res 2018;46:W296-w303.

63. Madeira F, Park YM, Lee J, Buso N, Gur T, Madhusoodanan N et al. The EMBL-EBI search and sequence analysis tools APIs in 2019. Nucleic Acids Res 2019;47:W636-W641.

64. Waterhouse AM, Procter JB, Martin DMA, Clamp M, Barton GJ. Jalview Version 2-a multiple sequence alignment editor and analysis workbench. Bioinformatics 2009;25:1189-1191.

65. Edgar RC. MUSCLE: multiple sequence alignment with high accuracy and high throughput. Nucleic Acids Res 2004;32:1792-1797.

66. Capella-Gutiérrez S, Silla-Martínez JM, Gabaldón T. trimAl: a tool for automated alignment trimming in large-scale phylogenetic analyses. Bioinformatics 2009;25:1972-1973.

67. Kumar S, Stecher G, Li M, Knyaz C, Tamura K. MEGA X: molecular evolutionary genetics analysis across computing platforms. Mol Biol Evol 2018;35:1547-1549.

68. Letunic I, Bork P. Interactive tree of life (iTOL) v3: an online tool for the display and annotation of phylogenetic and other trees. Nucleic Acids Res 2016;44:W242-W245.

69. Chaumeil P-A, Mussig A, Philip H, Parks D. GTDB-Tk: a toolkit to classify genomes with the Genome Taxonomy Database. Bioinformatics 2019;36:1925-1927.

70. Stamatakis A. RAxML version 8: a tool for phylogenetic analysis and post-analysis of large phylogenies. Bioinformatics 2014;30:1312-1313.

\section{Figures}




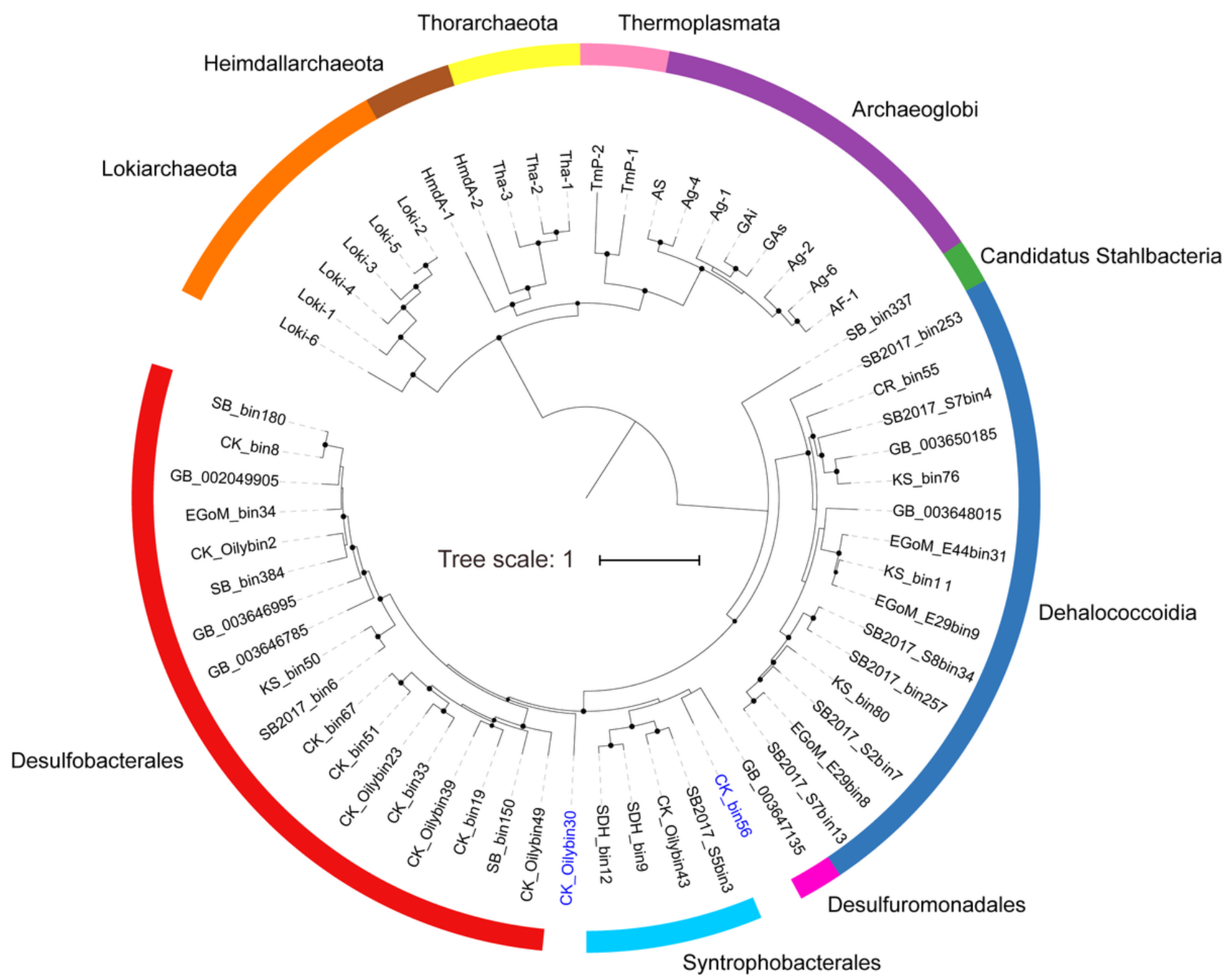

\section{Figure 1}

Classification of 62 genomes containing FaeA genes. The maximum-likelihood tree was built based on concatenation of 43 conserved protein sequences using RAxML with the PROTGAMMALG model. The scale bar represents one amino acid substitution per sequence position. Bootstrap values $>70 \%$ are indicated. Two genomess corresponding to unclassified orders within the Deltaproteobacteria are labelled blue. 


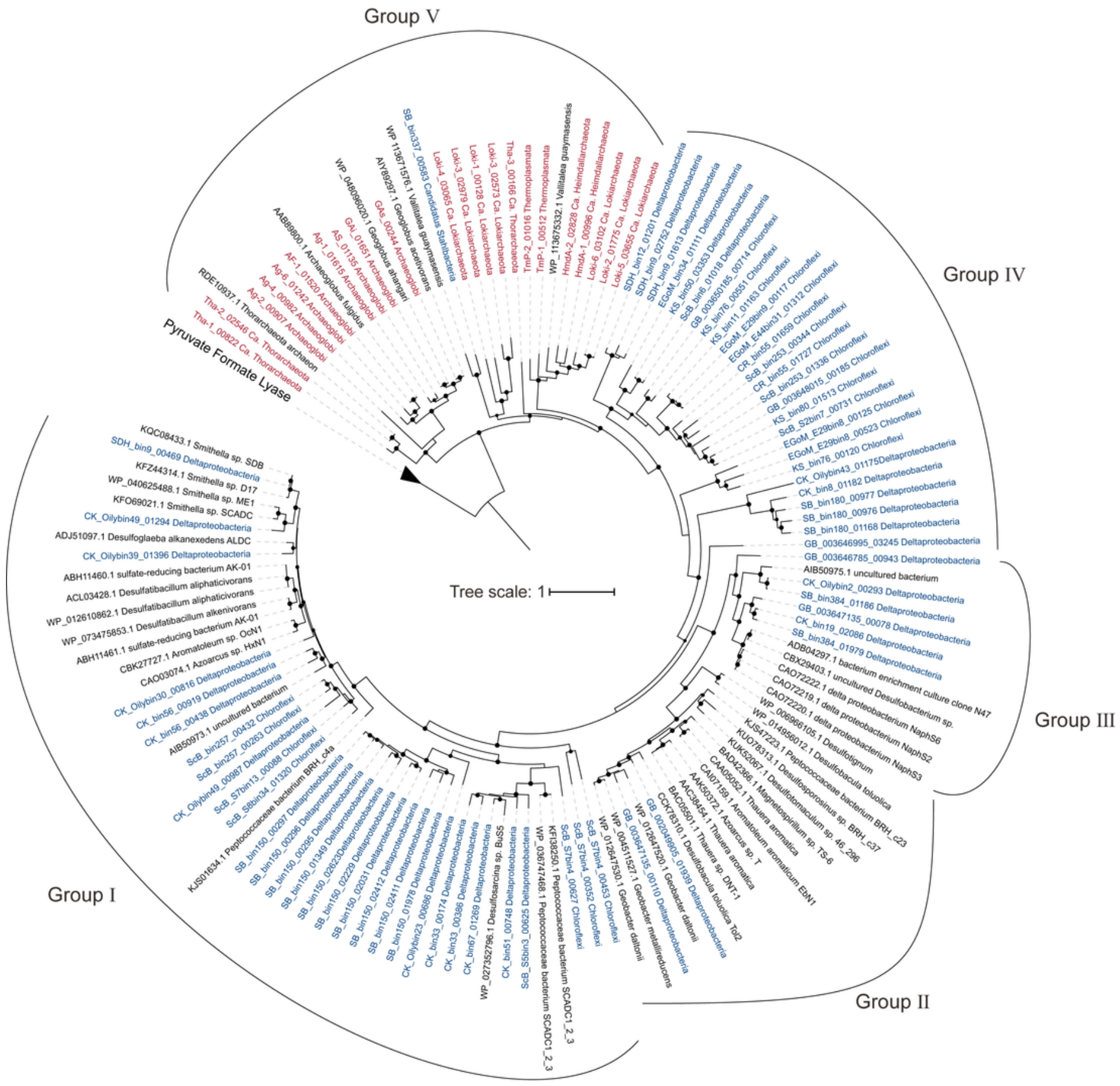

Figure 2

Phylogenetic affiliations of identified FaeA protein sequences. Alignments were generated with MUSCLE and trimmed using trimAl, and the tree was built using IQTree with the best-fit model and 1000 ultrafast bootstrap replicates. The scale bar represents one amino acid substitution per sequence position. Bootstrap values $>70 \%$ are indicated. Black: reference FaeA protein sequences; Blue: newly identified bacterial FaeA protein sequences in this study; Red: newly identified archaeal FaeA protein sequences in 
this study. The bacterial protein sequence in Group V (affiliated with Candidatus Stahlbacteria) could be contamination and was excluded from subsequent analyses.

a.

Dehalococcoidia CR_bin55 Dehalococcoidia EGoM E29bin8 Dehalococcoidia EGoM_E29bin9 Dehalococcoidia EGoM_E44bin31 Dehalococcoidia GB 003648015 Dehalococcoidia GB_003650185 Dehalococcoidia KS bin11 Dehalococcoidia KS_bin76 Dehalococcoidia KS bin80 Dehalococcoidia ScB_bin253 Dehalococcoidia ScB_bin257 Dehalococcoidia ScB S2bin7

Dehalococcoidia ScB_S 7 bin13

Dehalococcoidia ScB S7bin4 Dehalococcoidia ScB_S8bin34

Desulfobacterales $\mathrm{CK}$ bin8

Desulfobacterales CK_bin33

Desulfobacterales CK_bin51

Desulfobacterales CK bin67

Desulfobacterales CK_Oilybin2

Desulfobacterales CK Oilybin23

Desulfobacterales CK_Oilybin39

Desulfobacterales CK Oilybin49

Desulfobacterales EGoM_bin34

Desulfobacterales GB_002049905

Desulfobacterales GB_003646995

Desulfobacterales SB bin150

Desulfobacterales SB bin180

Desulfobacterales ScB_bin6

Deltaproteobacteria CK bin56

Deltaproteobacteria CK_Oilybin30

Syntrophobacterales ScB S5bin3

Syntrophobacterales SDH_bin9

Syntrophobacterales CK_Oilybin43

Syntrophobacterales SDH_bin12 Archaeoglobi AF-1 Archaeoglobi Ag-1 Archaeoglobi Ag-2 Archaeoglobi Ag-4 Archaeoglobi Ag-6 Archaeoglobi AS

Archaeoglobi GAi Archaeoglobi GAs Heimdallarchaeota HmdA-1 Heimdallarchaeota HmdA-2 Lokiarchaeota Loki-1 Lokiarchaeota Loki-2 Lokiarchaeota Loki-3 Lokiarchaeota Loki-4 Lokiarchaeota Loki-5 Lokiarchaeota Loki-6 Thorarchaeota Tha-1 Thorarchaeota Tha-2 Thorarchaeota Tha-3 Thermoplasmata TmP-1

Thermoplasmata TmP-2 Desulfobacterales CK bin19 Desulfobacterales KS bin50 Desulfobacterales GB_003646785 Desulfobacterales SB bin384 Desulfuromonadales GB_003647135<smiles>C1CC[As]C1</smiles>

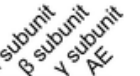

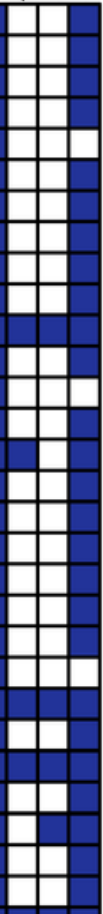

口-

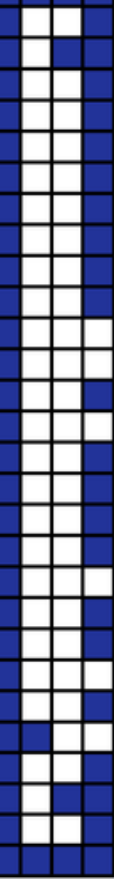

b.

C.

c.

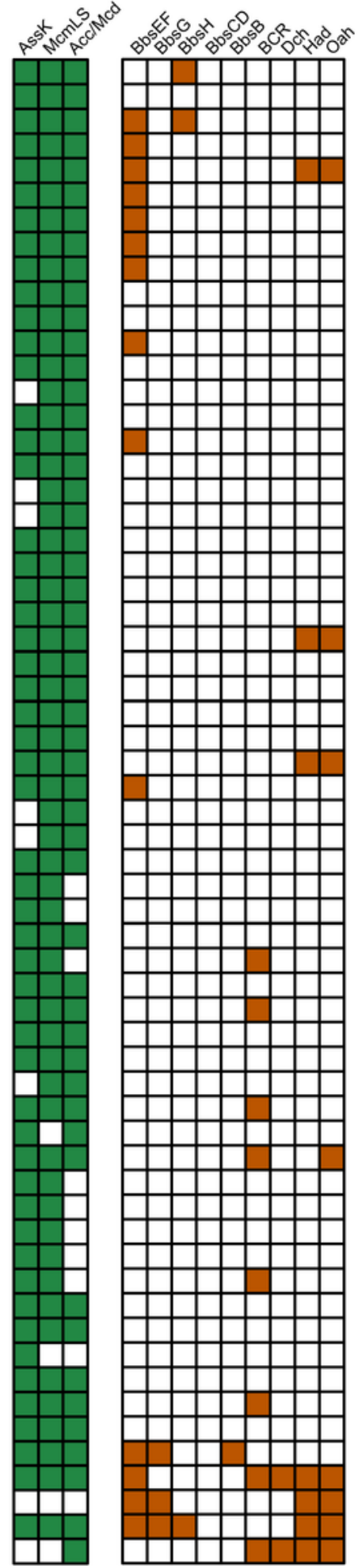

e.
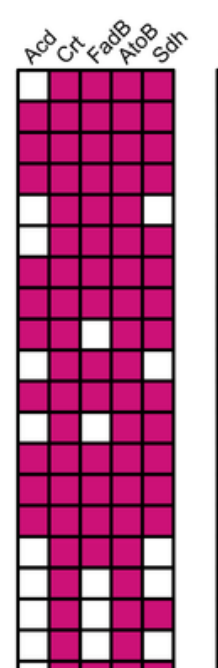

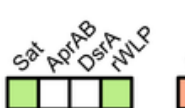
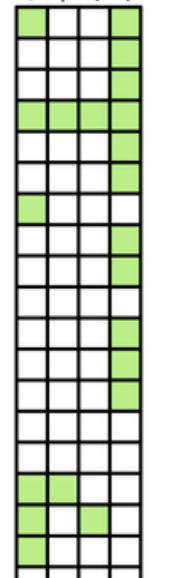
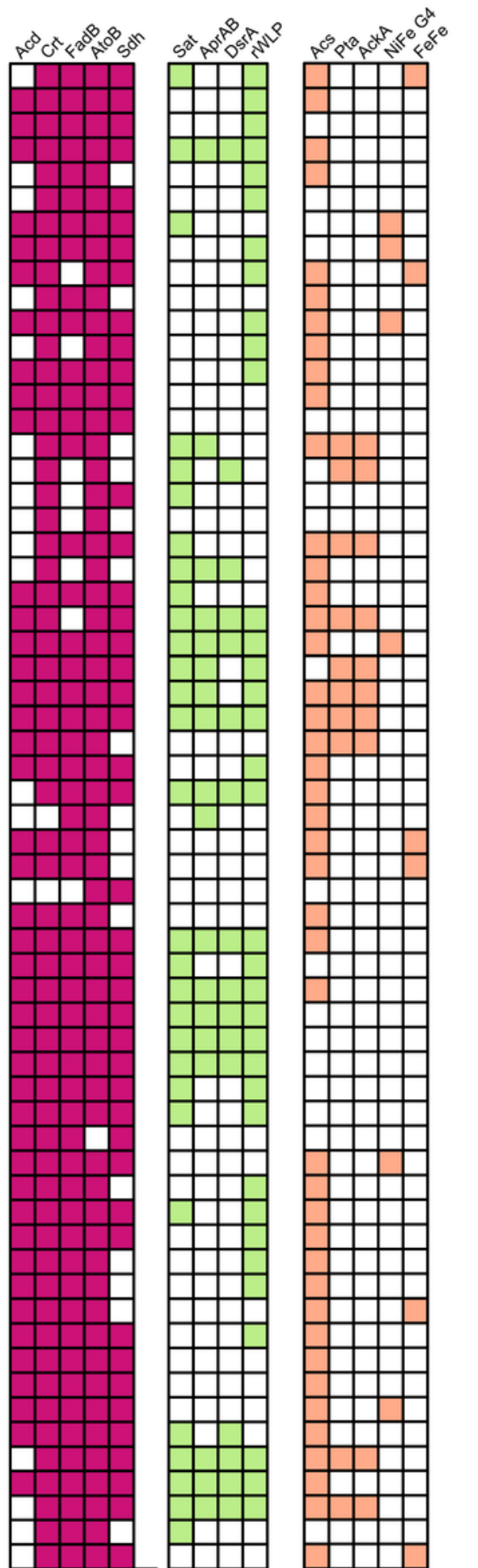

Figure 3

Identification of genes or pathways related to anaerobic hydrocarbon degradation. a, Alpha-, beta- and gamma- subunits and activating enzyme of FAE; b, Alkylsuccinate metabolism; c, Arylalkylsuccinate metabolism; $d, \beta$-oxidation and fumarate regeneration; e, Sulfate reduction and reverse Wood-Ljungdahl 
pathway; f, Fermentation. A pathway was considered to be present if at least half of the genes in this pathway were detected. Filled squares: presence of genes or pathways; Empty squares: absence of genes or pathways. Red shaded labels at left: genomes with potential to degrade aliphatic hydrocarbons; Blue shaded labels at left: genomes with potential to degrade alkyl-substituted monocyclic aromatic hydrocarbons. A complete list of metabolic information can be found in Table S7. Abbreviations: AE, activating enzyme; AssK, CoA-ligase; McmLS, methylmalonyl-CoA mutase; Acc, acetyl-CoA decarboxylase; Mcd, methylmalonyl-CoA decarboxylase; BbsEF, benzylsuccinate CoA-transferase; BbsG, benzylsuccinyl$\mathrm{CoA}$ dehydrogenase; $\mathrm{BbsH}$, phenylitaconyl-CoA hydratase; $\mathrm{BbsCD}$, 3-hydroxyacyl-CoA dehydrogenase; BbsB, benzoylsuccinyl-CoA thiolase; BcrA-D, benzoyl-CoA reductase; Dch, cyclohex-1,5-diene-1-carbonylCoA hydratase; Had, 6-hydroxycyclohex-1-ene-1-carbonyl-CoA dehydrogenase; Oah, 6-oxocyclohex-1-ene1-carbonyl-CoA hydrolase; Sdh, succinate dehydrogenase; Acd, acyl-CoA dehydrogenase; Crt, enoyl-CoA hydratases; FadB, 3-hydroxyacyl-CoA dehydrogenase; AtoB, acetyl-CoA acetyltransferase; Sat, sulfate adenylyltransferase; AprAB, adenylylsulfate reductase; DsrAB, dissimilatory sulfite reductase; Acs, acetylCoA synthetase (ADP-forming); Pta, phosphate acetyltransferase; AckA, acetate kinase; FeFe, FeFe hydrogenase; NiFe G4, Group4 NiFe hydrogenase.

a.

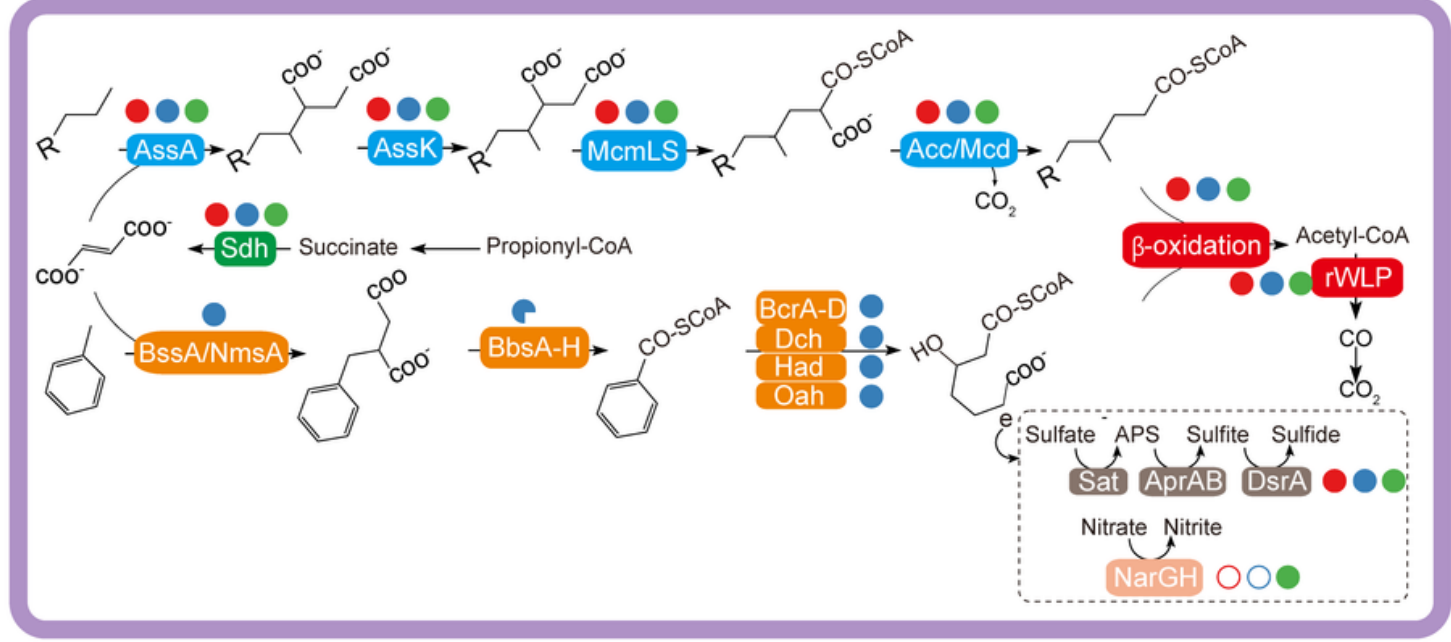

b.

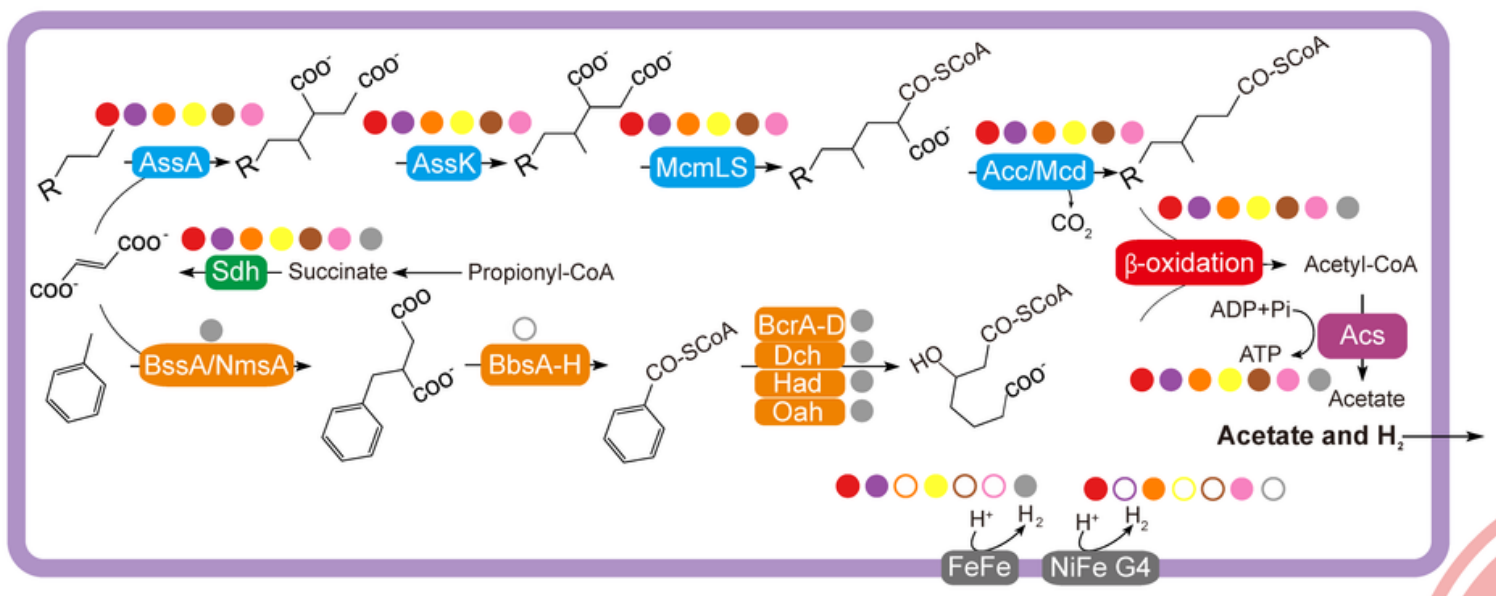

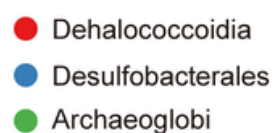
- Archaeoglobi - Syntrophobacterales - Heimdallarchaeota Lokiarchaeota - Thorarchaeota - Thermoplasmata Desulfuromonadales

Presence Absence - Partial subunits

\section{Figure 4}


Metabolic reconstruction of core pathways associated with anaerobic degradation of alkanes and alkylsubstituted monoaromatic hydrocarbons. a, Complete oxidation of hydrocarbons to $\mathrm{CO} 2$ through reverse Wood-Ljungdahl pathway coupled with reduction of an electron acceptor; $b$, Syntrophic hydrocarbon oxidation through interspecies transfer of hydrogen and acetate to methanogens. Gene abbreviations are indicated in Figure 3.

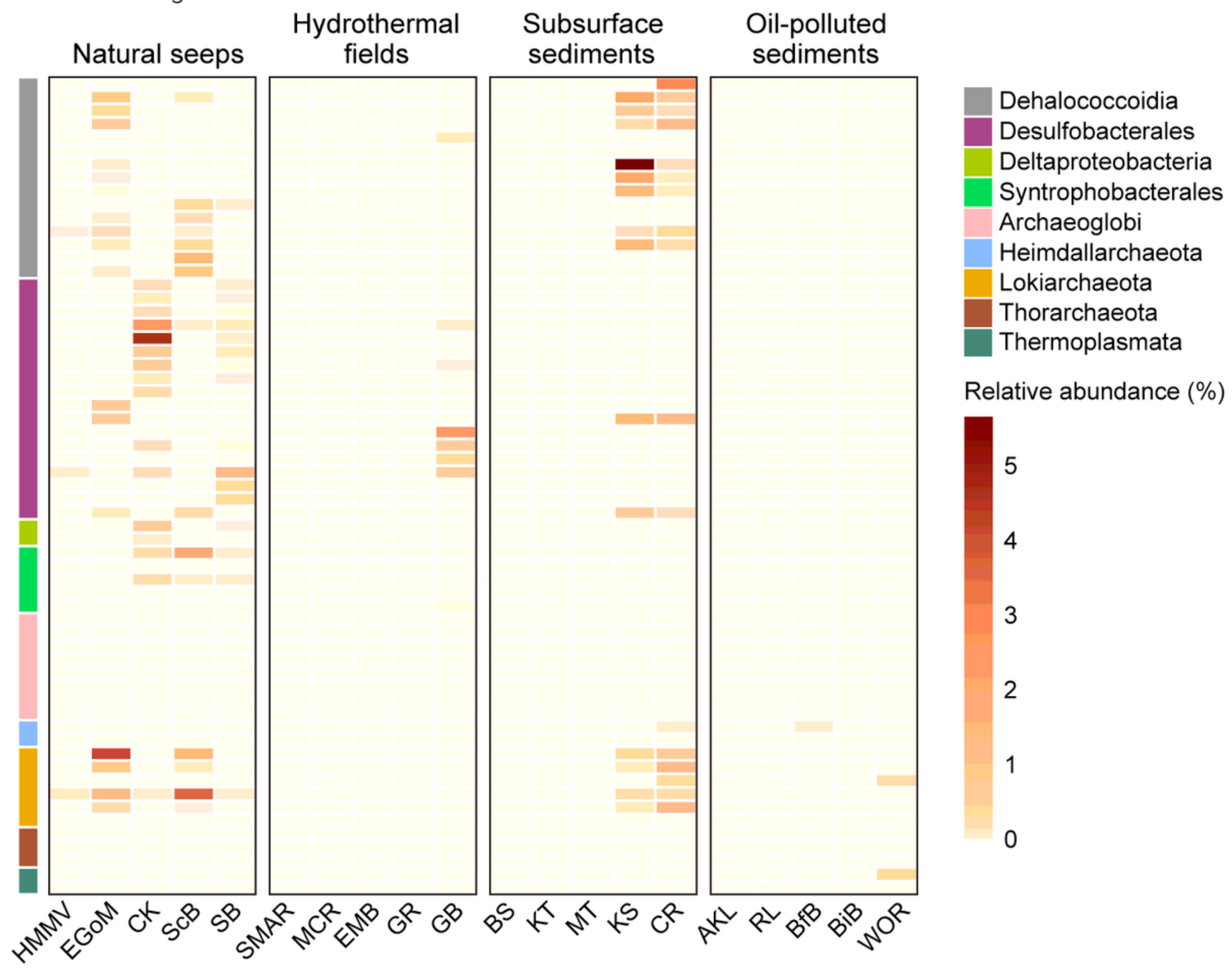

Figure 5

Biogeographic distribution of identified anaerobic hydrocarbon degraders across various marine sediment ecosystems. The 61 genomes are coloured according to their taxonomic lineages. Abbreviations: HMMV, Håkon Mosby Mud Volcano; EGoM, Eastern Gulf of Mexico; CK, Campeche Knolls; ScB, Scotain Basin; SB, Santa Barbara; SMAR, South Mid Atlantic Ridge; MCR, Mid-Cayman Rise; EMB, Eastern Manus Basin; GR, Gakkel Ridge; GB, Guaymas Basin; BS, Baltic Sea; KT, Kermadec Trench; MT, Mariana Trench; KS, Kattegat Sea; CR, Costa Rica; AKL, Al Kharrar Lagoon; RL, Rabigh Lagoon; BfB, Baffin Bay; BiB, Bioluminescent Bay; WOR, White Oak River estuary. Details on taxonomic assignments and relative abundances are in Tables S3 and S8, respectively. 


\section{Supplementary Files}

This is a list of supplementary files associated with this preprint. Click to download.

- DataS1.txt

- Figures5.pdf

- Figures4.pdf

- Figures2.pdf

- SupplementaryTables110.xlsx

- Supplementarylnformation.docx 